\title{
Funcionalidad familiar y conductas autolesivas en estudiantes, Institución Educativa José Carlos Mariátegui, Bongará, Amazonas
}

\section{Family functionality and self-injurious behaviors in students, José Carlos Mariátegui Educational Institution, Bongará, Amazonas}

\author{
Isabel Añazco Urbina ${ }^{1}$, Elito Mendoza Quijano
}

\section{RESUMEN}

La investigación tuvo como objetivo establecer la relación entre funcionalidad familiar y conductas autolesivas en estudiantes de la Institución Educativa José Carlos Mariátegui en la provincia de Bongará, región Amazonas, Perú. Se evaluó a 139 estudiantes del $1^{\circ}$ a $5^{\circ}$ de educación secundaria. Se aplicó el cuestionario de funcionalidad familiar (FFSIL) y la cédula de autolesión (CAL). Los resultados revelaron que, el 51,08\% de los estudiantes presentan ausencia de autolesión, $42,45 \%$ autolesión leve, $5,03 \%$ autolesión moderada y 1,44 autolesión grave, además el $55,4 \%$ tiene una familia moderadamente funcional, el $36,7 \%$ funcional, $6,5 \%$ disfuncional y $1.4 \%$ severamente funcional, el $27.3 \%$ de los estudiantes provenían de familia moderadamente funcional con ausencia de autolesión, $25.2 \%$ una familia moderadamente funcional con autolesión leve, $22.3 \%$ una familia funcional con ausencia de autolesión, con valores porcentaje más alto, mientras que el $0,7 \%$ tenía una familia gravemente disfuncional con autolesión leve, y el mismo porcentaje tenía una familia gravemente disfuncional sin autolesión siendo el porcentaje más bajo. Se concluye que existe una relación estadísticamente significativa entre funcionalidad familiar y las conductas autolesivas $\mathrm{p}=0.025<0.05$, además la fuerza de correlación es baja, Tau-b de Kendall $=$ 0.180 .

Palabras clave: Funcionalidad familiar, conductas autolesivas

\begin{abstract}
The objective of the research was to establish the relationship between family functionality and self-injurious behaviors in students of the José Carlos Mariátegui Educational Institution in the province of Bongará, Amazonas region, Peru. 139 students from 1 st to 5 th grade of secondary education were evaluated. The family functionality questionnaire (FFSIL) and the self-injury certificate (CAL) were applied. The results revealed that $51.08 \%$ of the students present absence of self-injury, $42.45 \%$ mild self-injury, 5.03\% moderate self-injury and 1.44 severe selfinjury, in addition $55.4 \%$ have a moderately functional family, 36.7\% functional, $6.5 \%$ dysfunctional and $1.4 \%$ severely functional, $27.3 \%$ of the students came from a moderately functional family with absence of self-injury, $25.2 \%$ a moderately functional family with mild self-injury, $22.3 \%$ a functional family with absence of self-injury, with higher percentage values, while $0.7 \%$ had a severely dysfunctional family with mild self-injury, and the same percentage had a severely dysfunctional family without self-injury being the lowest percentage. It is concluded that there is a statistically significant relationship between family functionality and self-injurious behaviors $\mathrm{p}=$ $0.025<0.05$, in addition the correlation strength is low, Kendall's Tau-b $=0.180$.
\end{abstract}

Keywords: Family functionality, self-injurious behaviors

\footnotetext{
${ }^{1}$ Bachiller en Psicología de la Facultad Ciencias de la Salud de la Universidad Nacional Toribio Rodríguez de Mendoza de Amazonas. Correo electrónico: isabelanascourbina@gmail.com.

${ }^{2}$ Maestro en gerencia y gestión en los servicios de salud. Docente de la Escuela Profesional de Enfermería. Facultad Ciencias de la Salud de la Universidad Nacional Toribio Rodríguez de Mendoza de Amazonas. Correo electrónico: elito.mendoza@untrm.edu.pe.
} 


\section{INTRODUCCIÓN}

Según la Organización Mundial de la Salud (OMS), (2017), más de un millón de adolescentes mueren cada año por causas prevenibles incluida la autolesión no suicida, que está considerada como la tercera causa de muertes en el mundo, señalando una alta tasa de mortalidad en Asia y Europa continental con cerca de 67.000 muertes de adolescentes.

En ese contexto, el Instituto Neurociencias del Hospital Clínico de Barcelona (2018), realizó un estudio para conocer el grado de conductas autolesivas en su población. El estudio reportó que el $4 \%$ de la población presentaba antecedentes de autolesiones; además destacó que estos comportamientos podrían tener consecuencias como lesiones personales, infecciones, hospitalización e incluso la muerte accidental. Asimismo, los resultados demostraron la prevalencia de conductas autolesivas en personas con edad entre 12 y 16 años, descartando la creencia que las autolesiones solo ocurren en personas con enfermedades mentales graves.

El estudio de caso realizado en la Universidad Técnica de Babahoyo, con la finalidad de investigar la dependencia existente entre disfuncionalidad en el hogar y las autolesiones, reportó que la influencia familiar es el componente más distinguido que influye negativamente en la conducta adolescente como consecuencia de la acumulación de experiencias tanto a nivel individual, familiar y social; determinando su forma de vida actual (Moreta, 2020).

Mayer (2016), en su trabajo "frecuencia de autolesiones e ideación suicida", en una población de alumnos del nivel secundario y preparatoria en México, evaluados mediante la cédula de indicadores para suicidas y la encuesta de maltrato infantil, las derivaciones probaron que un $27.9 \%$ de los estudiantes se autolesionaban y $7.4 \%$ aludieron tener ideación suicida, dejando observar mayor predominancia en las mujeres; relacionado a ello Díaz y Hurtado (2015), investigaron las conductas autolesivas (Cutting) en adolescentes de la Institución Educativa Padre Pio Sarobe - distrito de Santa Rosa de Ocopa, con la finalidad de determinar la presencia o ausencia de comportamientos autolesivos en los adolescentes. Los resultados demostraron que los adolescentes inmersos en conductas autolesivas, provenían de hogares disfuncionales donde las relaciones interpersonales dentro del ámbito familiar estaban en déficit.

Pontón (2018), investigó la disfuncionalidad familiar y su relación con el Cutting en una muestra constituida por 287 adolescentes del Colegio de Bachillerato Beatriz Cueva de Ayora, con la finalidad de determinar la relación entre variables. Los resultados mostraron que el $20.2 \%$ de los adolescentes evaluados se autolesionaban y el $48,28 \%$ provenían de una familia disfuncional leve, lo cual indica que la presencia de la disfuncionalidad familiar sí influye en la práctica de este tipo de conductas

Almeyda (2019), estudió la correspondencia entre violencia familiar y el cutting en jóvenes de I. E. del distrito de Comas. Los efectos mostraron una dependencia evidente entre la violencia familiar y el cutting $(\mathrm{p}<0.01)$, una correlación significativa entre la violencia familiar y las categorías de las conductas autolesivas $(\mathrm{p}<.01)$, así como una relación entre la práctica de autolesión y los niveles de violencia familiar $(\mathrm{p}<.01)$.

Niebles (2019), realizó un estudio en el que evaluó a 98 estudiantes de 12 y 18 años en el municipio de Soledad - Atlántico, con el propósito de conocer la relación entre disfuncionalidad familiar y Cutting, encontrando una correspondencia estadísticamente significativa entre variables al nivel de $<0,05$.

Aunque en los últimos años se está dando mayor importancia al tema de las conductas autolesivas y sus factores que lo originan, los estudios aún son escasos en el departamento de Amazonas y más aún en la provincia de Bongará, dónde se desconocen las estadísticas de presencia/ausencia de casos de autolesiones, que permitan visibilizar de manera más clara esta problemática.

\section{MATERIAL Y MÉTODO}

La presente investigación fue de tipo correlacional, no experimental (Hernández et al., 2014).

La población estuvo conformada por 473 alumnos del primer al quinto grado de la Institución Educativa José Carlos Mariátegui 2020. La muestra fue de 139 estudiantes. El muestreo fue probabilístico sistemático (Hernández et al., 2014).

Los instrumentos previamente validados y utilizados para la investigación fueron el cuestionario de funcionalidad familiar y la cédula de autolesiones (Canales, 1994), los cuales fueron aplicados de forma virtual, con el apoyo de tutores, padres de familia y auxiliares, en marzo del año 2021.

El cuestionario de funcionalidad familiar (FFSIL), fue validado por Álvarez (2018) cuyo nivel de confiabilidad fue de 0.883 y la cédula de autolesión (CAL), validado por Marín (2013) con un nivel de confiabilidad de 0.912 . 


\section{RESULTADOS}

Tabla 1

Frecuencia y porcentaje de estudiantes según edad y género.

\begin{tabular}{|c|c|c|c|c|c|c|}
\hline \multirow{3}{*}{$\begin{array}{l}\text { Edad } \\
\text { (Años) }\end{array}$} & \multicolumn{4}{|c|}{ Género } & & \\
\hline & \multicolumn{2}{|c|}{ Masculino } & \multicolumn{2}{|c|}{ Femenino } & \multicolumn{2}{|c|}{ Total } \\
\hline & fi & $\%$ & fi & $\%$ & fi & $\%$ \\
\hline 11 & 0 & 0,0 & 2 & $1,4 \%$ & 2 & 1,4 \\
\hline 12 & 2 & 1,4 & 2 & $1,4 \%$ & 4 & 2,9 \\
\hline \multirow[t]{2}{*}{13} & \multirow{2}{*}{27} & \multirow[t]{2}{*}{19,4} & 2 & 14,4 & \multirow[t]{2}{*}{47} & \multirow[t]{2}{*}{33,8} \\
\hline & & & 0 & $\%$ & & \\
\hline \multirow[t]{2}{*}{14} & \multirow{2}{*}{18} & \multirow[t]{2}{*}{12,9} & 2 & 16,5 & \multirow[t]{2}{*}{41} & \multirow[t]{2}{*}{29,5} \\
\hline & & & 3 & $\%$ & & \\
\hline \multirow[t]{2}{*}{15} & \multirow{2}{*}{12} & \multirow{2}{*}{8,6} & 1 & & \multirow[t]{2}{*}{24} & \multirow[t]{2}{*}{17,3} \\
\hline & & & 2 & 0,070 & & \\
\hline 16 & 6 & 4,3 & 9 & $6,5 \%$ & 15 & 10,8 \\
\hline 17 & 1 & 0,7 & 4 & $2,9 \%$ & 5 & 3,6 \\
\hline 18 & 1 & 0,7 & 0 & $0,0 \%$ & 1 & 0,7 \\
\hline \multirow[t]{2}{*}{ Total } & \multirow{2}{*}{67} & 48,2 & 7 & 51.8 & 13 & 100, \\
\hline & & $\%$ & 2 & & 9 & 0 \\
\hline
\end{tabular}

En la tabla 1, se puede apreciar que el 33,8\% de los estudiantes evaluados tenían 13 años de edad, de los cuales el $19.4 \%$ eran varones y el $14.4 \%$ eran mujeres, el 29,5\% de los adolescentes evaluados tenían 14 años de edad, de ellos $12.9 \%$ pertenecían al sexo masculino y $16.5 \%$ al sexo femenino, el $17,3 \%$ de los evaluados tenían 15 años de edad, de los cuales el $8.6 \%$ fueron varones y el $8.6 \%$ fueron mujeres.

Tabla2

Frecuencia y porcentaje según categorías de funcionalidad familiar de los estudiantes.

\begin{tabular}{lcc}
\hline \multicolumn{1}{c}{ Categorías } & fi & \% \\
\hline $\begin{array}{l}\text { Funcional } \\
\text { Moderadamente }\end{array}$ & 51 & 36,7 \\
funcional & 77 & 55,4 \\
$\begin{array}{l}\text { Disfuncional } \\
\text { Severamente } \\
\text { disfuncional }\end{array}$ & 9 & 6,5 \\
\hline \multicolumn{1}{c}{ Total } & $\mathbf{1 3 9}$ & 1,4 \\
\hline
\end{tabular}

En la tabla 2, se puede apreciar que el 36,7\% de los estudiantes evaluados procedían de una familia "funcional", seguido de un $55,4 \%$ con una familia "moderadamente funcional", un $6.5 \%$ con una familia "disfuncional y tan solo un $1.4 \%$ provenían de una familia "severamente disfuncional".

Tabla3

Frecuencia y porcentaje según categorías de conductas autolesivas de los estudiantes.

\begin{tabular}{lcc}
\hline \multicolumn{1}{c}{ Categorías } & fi & \% \\
\hline $\begin{array}{l}\text { Ausencia de } \\
\text { autolesión }\end{array}$ & 71 & 51,08 \\
$\begin{array}{l}\text { Autolesión leve } \\
\begin{array}{l}\text { Autolesión } \\
\text { moderada }\end{array}\end{array}$ & 79 & 42,45 \\
autolesión grave & 2 & 5,03 \\
\hline \multicolumn{1}{c}{ Total } & $\mathbf{1 3 9}$ & $\mathbf{1 0 0 , 0}$ \\
\hline
\end{tabular}

En la tala 3, respecto a las dimensiones de las autolesiones, los resultados evidenciaron que el $51.08 \%$ de los estudiantes de la Institución Educativa José Carlos Mariátegui presentaron "ausencia de autolesión", el 42,45\% ostentaron autolesión leve, el $5,03 \%$ mostraron "autolesión moderada" y un 1,43\% presentaron "autolesión grave".

Tabla4

Frecuencia y porcentaje según funcionalidad familiar y conductas autolesivas de los estudiantes.

\begin{tabular}{|c|c|c|c|c|c|c|c|c|c|c|}
\hline \multirow{3}{*}{$\begin{array}{c}\text { Funcionahidad } \\
\text { familiar }\end{array}$} & \multicolumn{8}{|c|}{ Conductas autolesivas } & \multirow{2}{*}{\multicolumn{2}{|c|}{ Total }} \\
\hline & \multicolumn{2}{|c|}{$\begin{array}{c}\text { Ausencia } \\
\text { de } \\
\text { autolesión }\end{array}$} & \multicolumn{2}{|c|}{$\begin{array}{c}\text { Autolesión } \\
\text { leve }\end{array}$} & \multicolumn{2}{|c|}{$\begin{array}{l}\text { Autolesión } \\
\text { moderada }\end{array}$} & \multicolumn{2}{|c|}{$\begin{array}{c}\text { Autolesión } \\
\text { grave }\end{array}$} & & \\
\hline & fi & $\%$ & $\mathrm{fi}$ & $\%$ & fi & $\%$ & fi & $\%$ & fi & $\%$ \\
\hline Funcional & 31 & 22,3 & 17 & 12,2 & 3 & 2,2 & 0 & 0,0 & 51 & 36,7 \\
\hline $\begin{array}{l}\text { Moderadamente } \\
\text { funcional }\end{array}$ & 38 & 27,3 & 35 & 25,2 & 2 & 1,4 & 2 & 1,4 & 77 & 55,4 \\
\hline Disfimcional & 1 & 0,7 & 6 & 4,3 & 2 & 1,4 & 0 & 0,0 & 9 & 6,5 \\
\hline $\begin{array}{l}\text { Severamente } \\
\text { disfincional }\end{array}$ & 1 & 0,7 & 1 & 0,7 & 0 & 0,0 & 0 & 0,0 & 2 & 1,4 \\
\hline Total & 71 & 51,1 & 59 & 42,4 & 7 & 5,0 & 2 & 1,4 & 139 & 100,0 \\
\hline
\end{tabular}

En la tabla 4, se observa que el $22,3 \%$ de los estudiantes evaluados tienen una familia funcional mostrando ausencia de autolesión; un 12,2\% tienen una familia funcional con presencia de autolesión leve; un $2,2 \%$ de los evaluados tienen una familia funcional con autolesión moderada, seguido de un $27,3 \%$ tienen una familia moderadamente funcional con ausencia de autolesión, un $25,2 \%$ tienen una familia moderadamente funcional con autolesión leve, un $1,4 \%$ tienen una familia moderadamente funcional con autolesión moderada, un 1,4 con familia moderadamente funcional y autolesión grave; un $0,7 \%$ de los evaluados tienen una familia disfuncional con ausencia de autolesión, un 4,3\% tienen una familia disfuncional con autolesión leve, 
un $1,4 \%$ tienen una familia disfuncional con autolesión moderada, un $0,7 \%$ de los adolescentes evaluados tiene una familia severamente disfuncional con ausencia de autolesión y un $0,7 \%$ tiene una familia severamente disfuncional con autolesión leve.

\section{Tabla5}

Prueba de hipótesis y correlación del nivel de funcionalidad familiar y las conductas autolesivas en los estudiantes.

\begin{tabular}{|c|c|c|c|c|c|}
\hline \multicolumn{6}{|c|}{ Medidas simétricas } \\
\hline & & Valor & $\begin{array}{c}\text { Error } \\
\text { estándar } \\
\text { asintótico }\end{array}$ & $\begin{array}{c}\mathrm{T} \\
\text { aproximada }\end{array}$ & $\begin{array}{c}\text { Significación } \\
\text { aproximada }\end{array}$ \\
\hline $\begin{array}{l}\text { Ordinal } \\
\text { por } \\
\text { ordinal }\end{array}$ & $\begin{array}{l}\text { Tau-b de } \\
\text { Kendall }\end{array}$ & 0,180 & 0,079 & 2,250 & 0,024 \\
\hline \multicolumn{2}{|c|}{$\begin{array}{l}\mathrm{N}^{\circ} \text { de casos } \\
\text { válidos }\end{array}$} & 139 & & & \\
\hline
\end{tabular}

En la tabla 5, se muestra que al aplicar la prueba estadística no paramétrica Tau-b de Kendall se obtuvo un valor de $0.180, \mathrm{p}=0.024$; por lo tanto, $\mathrm{p}=$ $0.024<0.05$ que es el nivel de significancia lo cual indica que hay una relación estadísticamente significativa entre el nivel de funcionalidad familiar y las conductas autolesivas en los estudiantes de la Institución Educativa José Carlos Mariátegui, así mismo se puede demostrar que la fuerza de correlación es débil, Tau-b de Kendall es 0,180.

\section{DISCUSIÓN}

De acuerdo a los resultados afirmamos que existe relación estadística significativa entre la funcionalidad familiar y las conductas autolesivas en los alumnos de la Institución Educativa José Carlos Mariátegui, entonces, cabe recalcar la importancia del factor familiar en el desarrollo y formación de cada individuo, por lo que es un área sensible en la adolescencia, etapa donde cada persona vive muchos cambios tanto a nivel biológico y psicológico, tiempo donde el sujeto ingresa a dificultades con su historia en sí, del mismo modo tiene que lidiar con la cultura y prejuicios de sus progenitores.

Garbarino, González \& Faragó (2010), hacen referencia a que la disfunción de los miembros al interior del grupo familiar puede crear un conjunto de conductas de peligro que van en contra la integridad de los adolescentes, siendo una de ellas el cutting.

La funcionalidad familiar en sus dimensiones de cohesión, adaptabilidad y comunicación es esencial en el desarrollo de los individuos y concluyente en la convivencia de sus integrantes, en este sentido es significativo lo encontrado, que del total de evaluados, el 36,7\% de los estudiantes procedían de una familia "funcional", seguido de un $55,4 \%$ con una familia "moderadamente funcional", un $6.5 \%$ con una familia "disfuncional y tan solo un $1.4 \%$ provenían de una familia "severamente disfuncional". De igual manera que en lo registrado (Pontón (2018) en su investigación, obtuvo que el $20,2 \%$ de los participantes se autolesionaban; investigando a fondo el porqué de esta práctica, se determinó que las motivaciones de estos adolescentes son la defectuosa relación con los padres $(28,10 \%)$, adolescentes que se autolesionan muestran en su mayoría una modalidad de familia Disfuncional Leve (48,3\%), seguido por el tipo de Familia Funcional $(37,9 \%)$ y en menor proporción Familia Disfuncional Grave (13,8\%).

Así mismo Caicedo, (2017) en su trabajo "Estrategias de intervención desde el trabajo social frente al cutting (auto laceraciones), demostró que la manifestación de práctica del cutting en su mayoría se debe a dificultades vividas dentro del sistema familiar: es decir el 39\% de los estudiantes se cortan debido a problemas familiares.

Con respecto a las conductas autolesivas, se entiende como toda actividad física que esté relacionada a herir a la persona que lo práctica de acuerdo a las dimensiones de las autolesiones, los resultados evidenciaron que el $51.1 \%$ de los estudiantes de la Institución Educativa José Carlos Mariátegui presentaron "ausencia de autolesión”, el 42,4\% ostentaron autolesión leve, el 5,0\% mostraron "autolesión moderada" y un 1,4\% presentaron "autolesión grave"; Díaz y Hurtado (2015), en su estudio: prácticas auto lesivas del cutting en estudiantes de la I. E. P. "Padre Pio Sarobe" del Distrito de Santa Rosa de Ocopa de la Provincia de Concepción- 2015, De los 50 estudiantes encuestados, 37 aceptan auto agredirse a través de cortes. Siguiendo los lineamientos políticos de salud mental, el Ministerio de Salud (2018), reporta que, en su mayoría los casos de prácticas autolesivas, se muestran a través de actos suicidas no explícitas, dentro de ellos el cutting tiene mayor prevalencia en zonas urbanas.

En este mismo sentido, Neyra (2015), en su investigación, "características de la violencia familiar y la autolesión en mujeres adolescentes", evidencia que existe una relación entre los niveles de violencia familiar y la autolesión. Coincidiendo con Pérez (2016), que estudió el "Funcionamiento familiar e ideación suicida de estudiantes de quinto de secundaria de instituciones educativas de un distrito de Lima" halló que existe una relación reveladora entre la disfunción familiar y mayor nivel de ideación suicida. Morante, L. (2016) en su trabajo, "análisis del cutting en los estudiantes del sexto año de la Escuela de Educación Básica Fiscal periodo 2015 - 2016" enfocándose en los ocasionales que llevan a la práctica del Cutting indica, que la escasa comunicación entre los adolescentes y sus padres son motivos principales que lleva a los alumnos a realizar la autolesión. Así también Díaz y Hurtado (2015), en 
su estudio manifiesta que, de los 50 estudiantes encuestados, 37 aceptan auto agredirse a través de cortes. Los motivos se relacionan en un 34\% (17) porque son golpeados por sus padres, 19\% (10), comparado con la presente investigación se puede apreciar la semejanza de resultados de la tabla 3, donde indica que $42,4 \%$ de los estudiantes presenta autolesión leve y un $4,3 \%$ de los ellos tienen una familia disfuncional.

Las derivaciones alcanzadas en la investigación sobre la funcionalidad y las conductas autolesivas en los estudiantes de la Institución Educativa José Carlos Mariátegui, indican que hay relación entre las variables con una significancia de $p=0.024<0.05$, sin embargo, es una correlación baja puesto que el valor de Tau-b de Kendall es 0,180; coinciden con los obtenidos por Urtecho (2017), en su trabajo: "Disfuncionalidad familiar relacionado a ideación no suicida en los adolescentes", donde encontró que existe una correlación positiva débil entre ambas variables, tras aplicar el correlacional de Spearman y obtener un $\mathrm{r}=0.185$ con una $\mathrm{p}=0.026$.

\section{CONCLUSIONES}

En la Institución Educativa José Carlos Mariátegui se puede evidenciar que existe dependencia entre las variables; funcionalidad familiar y conductas autolesivas, donde la fuerza de correlación es débil, donde Tau-b de Kendall $=0.180, \mathrm{p}=0,025<0.05$.

Concerniente a la variable funcionalidad familiar se encontró que de todos los estudiantes evaluados el mayor porcentaje de estudiantes, tienen una familia "funcional", seguido de "moderadamente funcional", continuo de "disfuncional" y un menor porcentaje de los estudiantes indicaron tener una familia "severamente disfuncional".

Según la cédula de autolesiones, los estudiantes de la Institución Educativa José Carlos Mariátegui se encuentran en las siguientes categorías de autolesión, un gran porcentaje indican no haberse autolesionado en su vida, $42,4 \%$ presenta autolesión leve, 5,0\% registra "autolesión moderada" y un solo un 1,4\% mencionaron haberse autolesionado gravemente.

De los 139 estudiantes que conformaron la muestra de estudio, el mayor porcentaje tenían la edad de 13 años, de los ellos/as la mayoría fueron del sexo masculino y el $14.4 \%$ del sexo femenino, el 29,5\% de los adolescentes evaluados tuvieron 14 años de edad, el menor porcentaje fueron hombres y $16.5 \%$ mujeres, por último, el 17,3\% de los evaluados con la edad de 15 años, donde el porcentaje tanto de hombres como de mujeres fue el mismo, alcanzando un $8,6 \%$

\section{REFERENCIAS BIBLIOGRÁFICAS}

Alberdi, I. (1999). La nueva familia española. Taurus: Madrid. http://www.marisol collazos.es/Sociologiacomplemento/Alberdi/ Resumen-la-nueva-familia-espanola.pdf.

Almeyda, C. (2020). Violencia familiar y autolesión en adolescentes de dos instituciones educativas del distrito de Comas, 2019. [tesis de grado, Universidad César Vallejo].

Apa. (2014). Manual diagnóstico y estadístico de los trastornos mentales DSM-5®. Editorial apa.

Caicedo Vargas, D. F. (2017). Estrategias de intervención desde el trabajo social frente al cutting (autolaceraciones) en el nivel básico de la Unidad Educativa Luis A. Martínez del cantón Ambato provincia de Tungurahua [Tesis de licenciatura, Universidad Técnica de Ambato,] Ecuador.

Canales, F.H., Alvarado, E.L. y Pineda, E.B. (1994). Metodología de la investigación: Manual para el desarrollo de personal de salud. (2da. Ed). Washington, EE. UU: Edit. Copyright.

Diaz, J., Hurtadi, C. (2015) El cutting en los estudiantes de la institución educativa Padre Pio Sarobe-distrito de santa rosa de ocopaprovincia de concepción. [tesis de grado, Universidad Nacional del centro del Perú].

Garbarino, J., Gónzalez, A., \& Faragó, P. (2010). Adolescencia y desarrollo emocional, socialy cognitivo. Saludy Bienestar de Adolescentes y Jóvenes: Una mirada integral, 55-59. http://publicaciones.ops.org.ar/publicaciones / otras \% $20 \mathrm{pub} / \mathrm{SaludBienestarAdol}$ escente.pdf

Hernández, R., Fernández, C. y Baptista, P. (2014). Metodología de la investigación. (6ta Ed) Editorial: McGraw-Hill, Interamericana. México.

Instituto de Neurociencias Hospital Clínico de Barcelona (2018). Revisión y actualización de la autolesión no suicida. España. Actas Esp Psiquiatric.

Marín, M. (2013). Desarrollo y evaluación de una terapia cognitivo conductual para adolescentes que se autolesionan. [tesis de grado, Universidad Nacional Autónoma de México]. Repositorio Institucional UNAM.

Mayer, P., et al. (2016). Adolescentes con autolesiones e ideación suicida: un grupo con mayor comorbilidad y adversidad 
psicosocial. Salud Pública de México, 58(3), 335- 336. https://dx.doi.org/10.21149/ spm.v58i3.7893

Ministerio de Salud (2018). Lineamientos de política sectorial de salud mental. Perú: Hecho el depósito legal de la biblioteca nacional.

Mora, R. (2016). Incidencia de la disfuncionalidad familiar en el comportamiento autolesivo no suicida de las estudiantes adolescentes [tesis de pregrado, Universidad de Guayaquil].

Morante, A. (2016). Análisis del Cutting en los Estudiantes del Sexto Año de la Escuela de Educación Básica Fiscal "División De Infantería $N^{\circ} 3$ Guayas" Periodo 2015 2016 [Tesis de licenciatura, Universidad de Guayaquil] Ecuador.

Moreta, B. (2020). Hogar disfuncional y su incidencia en el cutting en una estudiante [tesis de pregrado, Universidad Técnica de Babahoyo].

Neyra, N. (2015). Características de la Violencia Familiar y la Autolesión en mujeres adolescentes. [Tesis de licenciatura]. Perú. http://repositorio.ucv.edu.pe/bitstream/UCV /211/1/neyra_ae.pdf.

Niebles, J. V. (2019). Funcionalidad familiar en adolescentes con cutting: Asociación de las dimensiones de funcionalidad familiar $y$ práctica de cutting [tesis de Maestría, Universidad de la Costa].

Organización Mundial de la Salud (2017). Informe sobre la salud en el mundo 2014. Ginebra, Suiza: OMS.

Pérez, P. (2016). Funcionamiento familiar e ideación suicida de estudiantes de quinto de secundaria de instituciones educativas de un distrito de Lima. Universidad César Vallejo. Lima. Perú. http://repositorio.ucv.edu.pe/ bitstream/UCV/211/1/perez_ae.pdf

Pérez-Gonzales, E., De la Cuesta-Freijomil, D., Louro-Bernal, I. y Bayarre-Vea, H. (1997). Funcionamiento Familiar. Construcción y validación de un instrumento. Ciencias de la Salud Humana, 63-66.

Pontón, S. I. (2018). La disfunción familiar y su relación con el Cutting en adolescentes de 12 a 15 años del Colegio de Bachillerato Beatriz Cueva de Ayora en el periodo 2017-2018 [tesis de pregrado, Universidad Nacional de Loja].
Urtecho, A. (2017). Disfuncionalidad familiar relacionado a ideación suicida en los adolescentes. [tesis de grado, Universidad Privada Antenor Orrego]. Perú. 